VOL. 63 (2001) [321-327]

\title{
SOME NEW POINCARÉ-TYPE INEQUALITIES
}

\author{
Wing-Sum Cheung
}

\begin{abstract}
New and improved Poincaré-type integral inequalities involving many functions of many variables are established. These in turn can serve as generators and can generate numerous Poincaré-type integral inequalities by choosing different parameters.
\end{abstract}

\section{INTRODUCTION}

Poincaré's inequality is the integral inequality [12]

$$
\lambda_{0} \int_{\Omega} f^{2} d x \leqslant \int_{\Omega}|\nabla f|^{2} d x
$$

where $\Omega$ is a bounded region in $\mathbb{R}^{2}$ or $\mathbb{R}^{3}, f \in C^{1}(\Omega), f=0$ on the boundary of $\Omega$, and $\lambda_{0}$ is the smallest eigenvalue of the problem

$$
\begin{aligned}
\Delta f+\lambda f=0 & \text { in } \Omega, \\
f=0 & \text { on } \partial \Omega .
\end{aligned}
$$

Since (1) gives an effective estimate on the average of a $C^{1}$ function $f$ by the average of its gradient, it plays a fundamental role in the theory as well as applications of integral and differential equations. Variations and generalisations of (1) are generally known as Poincaré-type integral inequalities. A brief account on various Poincaré-type integral inequalities can be found in Beckenbach-Bellman [1], Hardy-Littlewood-Pólya [7], Milovanović-Mitrinović-Rassias [11], Mitrinović [12], Nirenberg [13], and more recently in Horgan et al $[\mathbf{8}, \mathbf{9}, \mathbf{1 0}]$, Pachpatte [14, 15], Rassias [16, 17], and Cheung [3]. It is the purpose of this paper to give some new and general Poincaré-type integral inequalities which improve the existing ones in the literature as special cases. It turns out that the method used here is very effective, easy to apply, and more importantly it is rather algorithmic in the sense that with slight modifications it can be applied to establishing other types of integral inequalities like Wirtinger-type integral inequalities

Received 26th June, 2000

The author is supported in part by a HKU CRCG grant.

The author wishes to thank the referee for his very thoughtful suggestions and comments.

Copyright Clearance Centre, Inc. Serial-fee code: 0004-9727/01 SA2.00+0.00. 
$[4,6]$, Sobolev-type integral inequalities $[2,4]$, and Opial-type integral inequalities [5], et cetera.

Throughout this paper $n \geqslant 1$ and $m \geqslant 2$ will be two fixed integers. For the sake of consistency we shall always take $i, j$ as indices running from 1 to $n$ and $\alpha, \beta$ from 1 to $m$. A general point in $\mathbb{R}^{n}$ is denoted by $x=\left(x_{1}, \ldots, x_{n}\right)$ and the volume form on $\mathbb{R}^{n}$ by $d x=d x_{1} \cdots d x_{n}$. If $\Omega$ is a region in $\mathbb{R}^{n}, C_{0}^{1}(\Omega)$ will denote, as usual, the collection of all real-valued continuously differentiable functions on $\Omega$ which vanish on the boundary $\partial \Omega$ of $\Omega$. Partial derivatives of $f \in C_{0}^{1}(\Omega)$ will be denoted by $f_{i}$, $i=1, \ldots, n$.

Since most summations and finite products appearing in this paper will be either from 1 to $n$ or from 1 to $m$, we shall simply write $\sum_{i=1}^{n}, \prod_{\alpha=1}^{m}, \ldots$ as $\sum_{i}, \prod_{\alpha}, \ldots$ when no confusion may arise.

The following elementary inequalities, which are easy consequences of the quadratic mean-arithmetic mean-geometric mean inequality, will be needed in the sequel (see, for example $[7,11,12])$.

LEMmA A. For any $p_{\alpha}>0$ and $q_{\alpha}, k_{\alpha} \geqslant 0$ satisfying $\sum q_{\alpha} / p_{\alpha}=1$,

$$
\prod_{\alpha} k_{\alpha}^{q_{\alpha}} \leqslant \sum_{\alpha} \frac{q_{\alpha}}{p_{\alpha}} k_{\alpha}^{p_{\alpha}}
$$

where the equality holds if and only if $k_{1}=\cdots=k_{m}$.

LEMma B. For any $r_{i} \geqslant 0$ and $s \geqslant 0$,

$$
\left(\sum_{i} r_{i}\right)^{s} \leqslant c(s, n) \sum_{i} r_{i}^{s}
$$

where

$$
c(s, n):= \begin{cases}n^{s-1} & \text { if } s \geqslant 1 \\ 1 & \text { if } 0 \leqslant s<1 .\end{cases}
$$

\section{MAIN RESULTS}

Let $\Omega=\prod_{i=1}^{n}\left[a_{i}, b_{i}\right]$ be a fixed rectangular region in $\mathbb{R}^{n}$, and $M:=\max \left\{b_{i}-a_{i}\right.$ : $i=1, \ldots, n\}$.

THEOREM 1. For any $f^{\alpha} \in C_{0}^{1}(\Omega)$ and any real numbers $p_{\alpha} \geqslant 2, q_{\alpha} \geqslant 0$ with $\sum_{\alpha} q_{\alpha} / p_{\alpha}=1$,

$$
\int_{\Omega} \prod_{\alpha}\left|f^{\alpha}\right|^{q_{\alpha}} \leqslant \frac{1}{n} \sum_{\alpha} \frac{q_{\alpha}}{p_{\alpha}}\left(\frac{M}{2}\right)^{p_{\alpha}} \int_{\Omega}\left|\nabla f^{\alpha}\right|^{p_{\alpha}}
$$


Theorem 1 generalises and improves some existing results of Poincaré-type inequalities in the literature $[\mathbf{3}, \mathbf{1 0}, \mathbf{1 2}, \mathbf{1 4}, \mathbf{1 5}]$. For example, we have the following simple consequences.

COROLLARY 2. [3] For any $f^{\alpha} \in C_{0}^{1}(\Omega)$ and any real numbers $p_{\alpha} \geqslant 2$ with $\sum_{\alpha} 1 / p_{\alpha}=1$,

$$
\int_{\Omega} \prod_{\alpha}\left|f^{\alpha}\right| \leqslant \frac{1}{n} \sum_{\alpha} \frac{1}{p_{\alpha}}\left(\frac{M}{2}\right)^{p_{\alpha}} \int_{\Omega}\left|\nabla f^{\alpha}\right|^{p_{\alpha}} .
$$

Proof: This follows immediately from Theorem 1 by letting $q_{\alpha}=1$ for all $\alpha$. $]$

Corollary 3. [3] For any $f^{\alpha} \in C_{0}^{1}(\Omega)$ and any real numbers $q_{\alpha} \geqslant 0$ with $q:=\sum_{\alpha} q_{\alpha} \geqslant 2$,

$$
\int_{\Omega} \prod_{\alpha}\left|f^{\alpha}\right|^{q_{\alpha}} \leqslant \frac{1}{n}\left(\frac{M}{2}\right)^{q} \sum_{\alpha} \frac{q_{\alpha}}{q} \int_{\Omega}\left|\nabla f^{\alpha}\right|^{q}
$$

Proof: This follows immediately from Theorem 1 by letting $p_{\alpha}=q$ for all $\alpha$. $\square$ Corollary 4. [3] For any $f^{\alpha} \in C_{0}^{1}(\Omega)$,

$$
\int_{\Omega} \prod\left|f^{\alpha}\right| \leqslant \frac{1}{n m} \sum_{\alpha}\left(\frac{M}{2}\right)^{m} \int_{\Omega}\left|\nabla f^{\alpha}\right|^{m} .
$$

Proof: This is immediate from Corollary 2 with $p_{\alpha}=m$ for all $\alpha$ or from Corollary 3 with $q_{\alpha}=1$ for all $\alpha$.

Note that from the preceding results, Poincaré-type inequalities involving only one function (the case $m=1$ ) can be obtained easily for free. For instance, we have

COROLlary 5. For any $f \in C_{0}^{1}(\Omega)$, and any real number $q \geqslant 2$,

$$
\int_{\Omega}|f|^{q} \leqslant \frac{1}{n}\left(\frac{M}{2}\right)^{q} \int_{\Omega}|\nabla f|^{q}
$$

Proof: This follows from Corollary 3 by letting $f^{\alpha}=f$ for all $\alpha$.

REMARK. Note that when $q=n=2$ and $\Omega$ is a square, the Poincaré-type integral inequality obtained in Corollary 5 above is sharper than that in [12]. In fact, here our constant is $M^{2} / 8$, while in [12] the constant is $7 M^{2} / 12$.

To prove Theorem 1 we need the following basic lemma.

Lemma 6 . If $f \in C_{0}^{1}(\Omega)$, then for any $t \in \Omega$,

$$
|f(t)| \leqslant \frac{1}{2 n} \sum_{i} \int_{a_{i}}^{b_{i}}\left|f_{i}\left(t_{1}, \ldots, t_{i-1}, u_{i}, t_{i+1}, \ldots, t_{n}\right)\right| d u_{i} .
$$


Proof: Since $f \in C_{0}^{1}(\Omega)$, for each $i=1, \ldots, n$, we have

$$
f(t)=\int_{a_{i}}^{t_{i}} f_{i}\left(t_{1}, \ldots, t_{i-1}, u_{i}, t_{i+1}, \ldots, t_{n}\right) d u_{i}
$$

and also

$$
f(t)=-\int_{t_{i}}^{b_{i}} f_{i}\left(t_{1}, \ldots, t_{i-1}, u_{i}, t_{i+1}, \ldots, t_{n}\right) d u_{i} .
$$

Taking absolute values and adding these up with respect to $i$, we then have

$$
2 n|f(t)| \leqslant \sum_{i} \int_{a_{i}}^{b_{i}}\left|f_{i}\left(t_{1}, \ldots, t_{i-1}, u_{i}, t_{i+1}, \ldots, t_{n}\right)\right| d u_{i}
$$

Proof of Theorem 1: By Lemma A, Lemma 6 and Lemma B, we have

$$
\begin{aligned}
\prod_{\alpha}\left|f^{\alpha}(t)\right|^{q_{\alpha}} & \leqslant \sum_{\alpha} \frac{q_{\alpha}}{p_{\alpha}}\left|f^{\alpha}(t)\right|^{p_{\alpha}} \\
& \leqslant \sum_{\alpha} \frac{q_{\alpha}}{p_{\alpha}}\left[\frac{1}{2 n} \sum_{i} \int_{a_{i}}^{b_{i}}\left|f_{i}^{\alpha}\right| d u_{i}\right]^{p_{\alpha}} \\
& \leqslant \sum_{\alpha} \frac{q_{\alpha}}{p_{\alpha}}\left(\frac{1}{2 n}\right)^{p_{\alpha}} c\left(p_{\alpha}, n\right) \sum_{i}\left(\int_{a_{i}}^{b_{i}}\left|f_{i}^{\alpha}\right| d u_{i}\right)^{p_{\alpha}}
\end{aligned}
$$

for all $t \in \Omega$. Since $p_{\alpha} \geqslant 1, c\left(p_{\alpha}, n\right)=n^{p_{\alpha}-1}$ and so

$$
\prod_{\alpha}\left|f^{\alpha}(t)\right|^{q_{\alpha}} \leqslant \frac{1}{n} \sum_{\alpha} \frac{q_{\alpha}}{p_{\alpha}}\left(\frac{1}{2}\right)^{p_{\alpha}} \sum_{i}\left(\int_{a_{i}}^{b_{i}}\left|f_{i}^{\alpha}\right| d u_{i}\right)^{p_{\alpha}}
$$

By Hölder's Inequality, we have

$$
\begin{aligned}
\prod_{\alpha}\left|f^{\alpha}(t)\right|^{q_{\alpha}} & \leqslant \frac{1}{n} \sum_{\alpha} \frac{q_{\alpha}}{p_{\alpha}}\left(\frac{1}{2}\right)^{p_{\alpha}} \sum_{i}\left[\left(b_{i}-a_{i}\right)^{\left(p_{\alpha}-1\right) / p_{\alpha}}\left(\int_{a_{i}}^{b_{i}}\left|f_{i}^{\alpha}\right|^{p_{\alpha}} d u_{i}\right)^{1 / p_{\alpha}}\right]^{p_{\alpha}} \\
& =\frac{1}{n} \sum_{\alpha} \frac{q_{\alpha}}{p_{\alpha}}\left(\frac{1}{2}\right)^{p_{\alpha}} M^{p_{\alpha}-1} \sum_{i} \int_{a_{i}}^{b_{i}}\left|f_{i}^{\alpha}\right|^{p_{\alpha}} d u_{i} .
\end{aligned}
$$

Now since

$$
\begin{aligned}
\sum_{i} \int_{\Omega} \int_{a_{i}}^{b_{i}}\left|f_{i}^{\alpha}\right|^{p_{\alpha}} d u_{i} d t & =\sum_{i} \int_{a_{i}}^{b_{i}} \int_{\Omega}\left|f_{i}^{\alpha}\right|^{p_{\alpha}} d t d u_{i} \\
& =\sum_{i}\left(\int_{\Omega}\left|f_{i}^{\alpha}\right|^{p_{\alpha}} d t\right)\left(b_{i}-a_{i}\right) \\
& \leqslant M \int_{\Omega}\left|f_{i}^{\alpha}\right|^{p_{\alpha}} d t
\end{aligned}
$$


we have

$$
\begin{aligned}
\int_{\Omega} \prod_{\alpha}\left|f^{\alpha}(t)\right|^{q_{\alpha}} d t & \leqslant \frac{1}{n} \sum_{\alpha} \frac{q_{\alpha}}{p_{\alpha}}\left(\frac{1}{2}\right)^{p_{\alpha}} M^{p_{\alpha}-1} \sum_{i} \int_{\Omega} \int_{a_{i}}^{b_{i}}\left|f_{i}^{\alpha}\right| d u_{i} d t \\
& \leqslant \frac{1}{n} \sum_{\alpha} \frac{q_{\alpha}}{p_{\alpha}}\left(\frac{1}{2}\right)^{p_{\alpha}} M^{p_{\alpha}} \int_{\Omega}\left|f_{i}^{\alpha}\right|^{p_{\alpha}} d t \\
& =\frac{1}{n} \sum_{\alpha} \frac{q_{\alpha}}{p_{\alpha}}\left(\frac{M}{2}\right)^{p_{\alpha}} \int_{\Omega}\left[\left(\sum_{i}\left|f_{i}^{\alpha}\right|^{p_{\alpha}}\right)^{2 / p_{\alpha}}\right]^{p_{\alpha} / 2} d t \\
& \leqslant \frac{1}{n} \sum_{\alpha} \frac{q_{\alpha}}{p_{\alpha}}\left(\frac{M}{2}\right)^{p_{\alpha}} \int_{\Omega}\left[c\left(\frac{2}{p_{\alpha}}, n\right) \sum_{i}\left(\left|f_{i}^{\alpha}\right|^{p_{\alpha}}\right)^{2 / p_{\alpha}}\right]^{p_{\alpha} / 2} d t
\end{aligned}
$$

by Lemma B. Since $p_{\alpha} \geqslant 2, c\left(\left(2 / p_{\alpha}\right), n\right)=1$ for all $\alpha$ and so

$$
\int_{\Omega} \prod_{\alpha}\left|f^{\alpha}(t)\right|^{q_{\alpha}} d t \leqslant \frac{1}{n} \sum_{\alpha} \frac{q_{\alpha}}{p_{\alpha}}\left(\frac{M}{2}\right)^{p_{\alpha}} \int_{\Omega}\left|\nabla f^{\alpha}\right|^{p_{\alpha}} d t
$$

THEOREM 7. For any $f^{\alpha} \in C_{0}^{1}(\Omega)$ and any real numbers $p_{\alpha} \geqslant 2, q_{\alpha} \geqslant 0$ with $\sum q_{\alpha} / p_{\alpha}=1$,

$$
\int_{\Omega} \sum_{\beta}\left(\prod_{\alpha \neq \beta}\left|f^{\alpha}\right|^{q_{\alpha}}\right)\left|\nabla f^{\beta}\right|^{q_{\beta}} \leqslant K(p, q) \sum_{\alpha} \frac{q_{\alpha}}{p_{\alpha}} \int_{\Omega}\left|\nabla f^{\alpha}\right|^{p_{\alpha}}
$$

where

$$
K(p, q)=K\left(p_{\alpha}, q_{\alpha}\right):=\sum_{\beta}\left(\frac{1}{n}\right)^{1-\left(q_{\beta} / p_{\beta}\right)}\left(\frac{M}{2}\right)^{\sum_{\alpha \neq \beta} q_{\alpha}} .
$$

Theorem 7 generalises and improves some existing results of Poincaré-type integral inequalities in the literature $[\mathbf{3}, \mathbf{1 0}, \mathbf{1 2}, \mathbf{1 4}, \mathbf{1 5}]$. The following are simple consequences of Theorem 7.

COROLlaRY 8. [3] For any $f^{\alpha} \in C_{0}^{1}(\Omega)$ and any real numbers $p_{\alpha} \geqslant 2$ satisfying $\sum_{\alpha} 1 / p_{\alpha}=1$,

$$
\int_{\Omega} \sum_{\beta}\left(\prod_{\alpha \neq \beta}\left|f^{\alpha}\right|\right)\left|\nabla f^{\beta}\right| \leqslant\left(\frac{M}{2}\right)^{m-1}\left[\sum_{\alpha}\left(\frac{1}{n}\right)^{1-\left(1 / p_{\alpha}\right)}\right]\left[\sum_{\alpha} \frac{1}{p_{\alpha}} \int_{\Omega}\left|\nabla f^{\alpha}\right|^{p_{\alpha}}\right] .
$$

Proof: This follows immediately from Theorem 7 by putting $q_{\alpha}=1$ for all $\alpha$. 0 Corollary 9. [3] For any $f^{\alpha} \in C_{0}^{1}(\Omega)$,

$$
\int_{\Omega} \sum_{\beta}\left(\prod_{\alpha \neq \beta}\left|f^{\alpha}\right|\right)\left|\nabla f^{\beta}\right| \leqslant\left(\frac{M}{2}\right)^{m-1}\left(\frac{1}{n}\right)^{1-(1 / m)} \sum_{\alpha} \int_{\Omega}\left|\nabla f^{\alpha}\right|^{m}
$$


Proof: This is a simple consequence of Corollary 8 by putting $p_{\alpha}=m$ for all $\alpha$.

ProOF OF THEOREM 7: By a generalisation of Hölder's inequality for the case of many functions and Corollary 5 , we have

$$
\begin{aligned}
& \int_{\Omega} \sum_{\beta}\left(\prod_{\alpha \neq \beta}\left|f^{\alpha}\right|^{q_{\alpha}}\right)\left|\nabla f^{\beta}\right|^{q_{\beta}} \\
& \leqslant \sum_{\beta}\left\{\prod_{\alpha \neq \beta}\left[\int_{\Omega}\left|f^{\alpha}\right|^{p_{\alpha}}\right]^{q_{\alpha} / p_{\alpha}} \cdot\left[\int_{\Omega}\left|\nabla f^{\beta}\right|^{p_{\beta}}\right]^{q_{\beta} / p_{\beta}}\right\} \\
& \quad \leqslant \sum_{\beta}\left\{\prod_{\alpha \neq \beta}\left[\frac{1}{n}\left(\frac{M}{2}\right)^{p_{\alpha}} \int_{\Omega}\left|\nabla f^{\alpha}\right|^{p_{\alpha}}\right]^{q_{\alpha} / p_{\alpha}} \cdot\left[\int_{\Omega}\left|\nabla f^{\beta}\right|^{p_{\beta}}\right]^{q_{\beta} / p_{\beta}}\right\} \\
& =\left[\sum_{\beta}\left(\frac{1}{n}\right)^{\sum_{\alpha \neq \beta} q_{\alpha} / p_{\alpha}}\left(\frac{M}{2}\right)^{\sum_{\alpha \neq \beta} q_{\alpha}}\right] \cdot \prod_{\alpha}\left[\int_{\Omega}\left|\nabla f^{\alpha}\right|^{p_{\alpha}}\right]^{q_{\alpha} / p_{\alpha}} \\
& =K(p, q) \cdot \prod_{\alpha}\left[\int_{\Omega}\left|\nabla f^{\alpha}\right|^{p_{\alpha}}\right]^{q_{\alpha} / p_{\alpha}} .
\end{aligned}
$$

Therefore, by Lemma A, we conclude that

$$
\int_{\Omega} \sum_{\beta}\left(\prod_{\alpha \neq \beta}\left|f^{\alpha}\right|^{q_{\alpha}}\right)\left|\nabla f^{\beta}\right|^{q_{\beta}} \leqslant K(p, q) \cdot \sum_{\alpha} \frac{q_{\alpha}}{p_{\alpha}} \int_{\Omega}\left|\nabla f^{\alpha}\right|^{p_{\alpha}} .
$$

REMARK. Further interesting Poincaré-type integral inequalities can easily be generated by the results above. For instance, by taking $m=3$ in Corollary 9 , we have

$$
\int_{\Omega}|f g \nabla h+g h \nabla f+h f \nabla g| \leqslant \frac{M^{2}}{4 n^{2 / 3}}\left[\int_{\Omega}|\nabla f|^{3}+\int_{\Omega}|\nabla g|^{3}+\int_{\Omega}|\nabla h|^{3}\right]
$$

taking $m=2$ in Corollary 9 , we have

$$
\int_{\Omega}|f \nabla g+g \nabla f| \leqslant \frac{M}{2 \sqrt{n}}\left[\int_{\Omega}|\nabla f|^{2}+\int_{\Omega}|\nabla g|^{2}\right]
$$

and by putting $f=g=h$ in these inequalities, we obtain

$$
\int_{\Omega} f^{2}|\nabla f| \leqslant \frac{M^{2}}{4 n^{2 / 3}} \int_{\Omega}|\nabla f|^{3}
$$

and

$$
\int_{\Omega}|f \nabla f| \leqslant \frac{M}{2 \sqrt{n}} \int_{\Omega}|\nabla f|^{2}
$$




\section{REFERENCES}

[1] E.F. Beckenbach and R. Bellman, Inequalities (Springer-Verlag, Berlin, Heidelberg, New York, 1965).

[2] W.S. Cheung, 'On integral inequalities of the Sobolev type', Aequationes Math. 49 (1995), 153-159.

[3] W.S. Cheung, 'On Poincaré-type integral inequalities', Proc. Amer. Math. Soc. 119 (1993), 857-863.

[4] W.S. Cheung, 'Some multi-dimensional integral inequalities', in Nonlinear Mathematical Analysis and Its Applications, (Th.M. Rassias, Editor) (Hadronic Press, Palm Harbor, 1998), pp. 19-38.

[5] W.S. Cheung, 'Some new Opial-type inequalities', Mathematika 37 (1990), 136-142.

[6] W.S. Cheung, Z. Hanjš and J. Pečarić, 'On Wirtinger-type integral inequalities', (preprint).

[7] G.H. Hardy, J.E. Littlewood and G. Pólya, Inequalities (Cambridge Univ. Press, Cambridge, 1952).

[8] C.O. Horgan, 'Integral bounds for solutions of nonlinear reaction-diffusion equations', $Z$. Angew. Math. Phys. 28 (1977), 197-204.

[9] C.O. Horgan and R.R. Nachlinger, 'On the domain of attraction for steady states in heat conduction', Internat. J. Engrg. Sci. 14 (1976), 143-148.

[10] C.O. Horgan and L.T. Wheeler, 'Spatial decay estimates for the Navier-Stokes equations with applications to the problem of entry flow', SIAM J. Appl. Math. 35 (1978), 97-116.

[11] G.V. Milovanović, D.S. Mitrinović and Th.M. Rassias, Topics in polynomials: Extremal problems, inequalities, zeros (World Scientific Publishing Co., River Edge, N.J., 1994).

[12] D.S. Mitrinović, Analytic inequalities (Springer-Verlag, Berlin, Heidelberg, New York, 1970).

[13] L. Nirenberg, 'On elliptic partial differential equations', Ann. Scuola Norm. Sup. Pisa Cl. Sci. (4) 13 (1959), 116-162.

14] B.G. Pachpatte, 'On Poincaré type integral inequalities', J. Math. Anal. Appl. 114 (1986), 111-115.

[15] B.G. Pachpatte, 'On some new integral inequalities in several independent variables', Chinese J. Math. 14 (1986), 69-79.

[16] Th.M. Rassias, 'On certain properties of eigenvalues and the Poincaré inequality', in Global Analysis - Analysis on Manifolds, Teubner-Texte zur Math 57 (Teubner, Leipzig, 1983), pp. 282-300.

[17] Th.M. Rassias, 'Un contre-exemple à l'inégalité de Poincaré', C.R. Acad. Sciences Paris 284 (1977), 409-412.

\footnotetext{
Department of Mathematics

The University of Hong Kong

Hong Kong

e-mail: wscheung@hkucc.hku.hk
} 